\title{
Awareness, knowledge, and practice patterns of general practitioner residents and specialists toward hidradenitis suppurativa: a survey study
}

Sofia Lopes ${ }^{1 凶}$, Júlia Vide ${ }^{1}$, Miguel Costa-Silva ${ }^{1}$, Filomena Azevedo ${ }^{1}$, Sofia Magina ${ }^{1,2}$

\begin{abstract}
Introduction: Hidradenitis suppurativa (HS) is an unrecognized chronic inflammatory and debilitating disease with severe consequences for patients' quality of life.

Methods: A survey was performed among general practitioner (GP) residents and consultants in order to determine awareness, knowledge, and attitudes about HS.

Results: Among 372 respondents, $74 \%$ were GP residents in the first 2 years, $22 \%$ GP residents in the 3 rd and 4 th year, and $4 \%$ consultants. For a patient with boils and/or recurrent abscesses in folds, $90 \%$ considered a diagnosis of HS with no significant difference according to years of experience. These patients were referred to dermatology by 273 residents ( $80 \%$ ) and eight consultants $(53 \%)$, and this difference is statistically significant $(p<0.05)$. Regarding acute treatment, $84 \%$ prescribed topical antibiotics and $76 \%$ oral antibiotics. Respecting therapeutic approach, we observed that treatment with non-steroidal anti-inflammatory drugs is higher among older residents $(51 \%)$ compared to younger ones $(36 \%, p<0.02)$ and the prescription of oral clindamycin is higher among consultants $(31 \%)$ compared to residents ( $12 \%, p<0.04)$.

Conclusions: Our survey demonstrates that knowledge of HS is lacking among primary care physicians. Communication channels between GPs and dermatologists are often hampered, and so we recommend incorporating medical education into GP residency programs on how to treat mild HS, when to refer, and how to approach HS.
\end{abstract}

Keywords: hidradenitis suppurativa, general practitioners, awareness, survey

Received: 6 January 2019 | Returned for modification: 19 January 2019| Accepted: 28 January 2019

\section{Introduction}

Hidradenitis suppurativa (HS) is a chronic, recurrent, inflammatory skin disease, characterized by the presence of painful nodules and abscesses in apocrine gland-bearing areas (1-3). The rupture of these lesions leads to the formation of sinus tracts and scarring, causing progressive worsening of the clinical prognosis $(1,2)$. Furthermore, affected areas are frequently malodourous with recurrent purulent discharge (3). As a consequence, patients with HS experience a substantial loss of quality of life $(1,4)$.

Awareness of HS is still lacking among physicians, contributing to delayed diagnosis or, in extreme cases, to misdiagnosis $(1,5)$. These gaps result in more advanced-stage patients, whose therapeutic options become progressively limited.

The primary aim of this study was to assess screening practices and awareness among general practitioner (GP) residents and consultants regarding HS characteristics and therapeutic approaches.

\section{Methods}

With the permission of Parsi et al. (6) and Costa-Silva et al. (7), we adapted a questionnaire and then distributed it to all GP residents and consultants attending two dermatology meetings dedicated to GPs in December 2015 and April 2016. Epidemiology, pathophysiology, diagnostic, and management questions regarding HS were adjusted and gathered together in this questionnaire.

For the results analysis, the participants were divided into three groups: GP residents in the first 2 years of residency, GP residents in the last 2 years of residency, and GP consultants. All statistical analyses were performed using SPSS version 20.0 (IBM, Armonk, NY). Categorical variables were compared using Fisher's exact test or a chi-square test. We considered the significance level to be $p<0.05$.

\section{Results}

The overall response rate was $62 \%$ (366 of 593), with 283 women $(77 \%)$. Among the respondents, 187 were GP residents in their 1st year (51\%), 84 in their 2nd year (23\%), 58 in their 3rd year (16\%), 21 in their 4 th year $(6 \%)$, and 16 were consultants ( $4 \%$; Table 1$)$. For a patient with boils and/or recurrent cysts located at folds, 327 considered a diagnosis of HS (90\%), whereas 35 did not (10\%), with no significant difference in the responses according to the years of experience $(p=0.429)$. In addition, $169(46 \%)$ reported evaluating

Table 1 | Demographic and practice characteristics of GP respondents.

\begin{tabular}{lc}
\hline Variable & GP respondents, $n(\%)$ \\
\hline Sex & \\
Male & $85(23)$ \\
Female & $283(77)$ \\
Age (years) & \\
$<30$ & $298(81)$ \\
$30-35$ & $51(14)$ \\
$>35$ & $18(5)$ \\
Residency year & \\
1 st & $187(51)$ \\
2 nd & $84(23)$ \\
3rd & $58(16)$ \\
4th & $21(6)$ \\
Consultants & $16(4)$
\end{tabular}

$\mathrm{GP}=$ general practitioner. 
one to five patients per month with these characteristics. Concerning factors associated with HS, 73\% associated it with hyperhidrosis, $71 \%$ with friction, $64 \%$ with hormonal dysfunction, $47 \%$ with obesity, $46 \%$ with acne, $44 \%$ with diabetes mellitus, $34 \%$ with systemic inflammation, $25 \%$ with smoking, $23 \%$ with alcoholism, $12 \%$ with depression, and $6 \%$ with inflammatory bowel disease (IBD), with no significant differences observed between GP degree ( $p>0.05$; Table 2). These types of patients were referred to dermatology consultation by 273 residents ( $80 \%)$, but only by eight consultants (53\%), and this difference is statistically significant $(p=0.01)$. General surgery, plastic surgery, and gynecology are other specialties to which these patients are directed (at $36 \%$, $5 \%$, and $2 \%$ respectively). Among these, $67 \%$ of the consultants referred patients for general surgery against only $35 \%$ of the residents, and the differences are statistically significant $(p=0.01)$.

Table 2 | Conditions in HS patients that GP respondents consider important for investigation.

\begin{tabular}{lc}
\hline Condition & $\begin{array}{c}\text { No. and percentage (\%) of GPs } \\
\text { responding positively }\end{array}$ \\
\hline Acne & $169(46)$ \\
Obesity & $194(53)$ \\
Diabetes mellitus & $159(44)$ \\
Hormonal disorders & $234(64)$ \\
Systemic inflammation & $127(35)$ \\
IBD & $22(6)$ \\
Depression & $44(12)$ \\
Smoking & $89(25)$ \\
Alcoholism & $85(23)$ \\
Hyperhidrosis & $266(73)$ \\
Friction & $258(71)$ \\
Cutaneous infection & $310(85)$ \\
None of these & $3(1)$ \\
\hline
\end{tabular}

$\mathrm{IBD}=$ inflammatory bowel disease, GPs = general practitioners.

Table 3 | Common HS treatments used by GP respondents.

\begin{tabular}{lc}
\hline & $\begin{array}{c}\text { No. and percentage (\%) of GPs } \\
\text { responding positively }\end{array}$ \\
\hline Drainage & $211(59)$ \\
NSAIDs & $140(39)$ \\
Oral contraceptives & $31(9)$ \\
Topical antibiotics & $303(84)$ \\
Clindamycin & $43(12)$ \\
Erythromycin & $15(4)$ \\
Fusidic acid & $277(77)$ \\
Mupirocin & $53(15)$ \\
Systemic antibiotics & $273(76)$ \\
Betalactams & $191(53)$ \\
Clindamycin & $48(13)$ \\
Tetracyclines & $38(11)$ \\
Rifampicin & $4(1)$ \\
Macrolides & $24(7)$ \\
Fluoroquinolones & $47(13)$ \\
Retinoids & \\
Topic & $26(7)$ \\
Systemic & $6(2)$ \\
Corticosteroids & \\
Topic & $78(24)$ \\
Intralesional & $7(2)$ \\
Systemic & $10(3)$ \\
\hline NSAIDs = non-steroidal anti-inflammatory drugs, GPs & general practitioners.
\end{tabular}

Finally, regarding acute HS treatment, 39\% of the respondents prescribed non-steroidal anti-inflammatory drugs (NSAIDs), 59\% abscess drainage, $9 \%$ oral contraceptives, $84 \%$ topical antibiotics, $7 \%$ topical retinoids, $2 \%$ oral retinoids, $76 \%$ oral antibiotics, $24 \%$ topical steroids, and $2 \%$ oral steroids (Table 3 ). Regarding the therapeutic approach, we determined the following statistically significant differences among young residents, advanced residents, and consultants: treatment with NSAIDs is higher among older residents $(51 \%)$ compared to younger ones $(36 \% ; p=0.02)$, and the prescription of oral clindamycin is higher among consultants (31\%) compared to residents $(12 \% ; p=0.047$; Table 4).

\section{Discussion}

Until recently, HS was considered an orphan disease because it was treated by several specialties, although it was assigned to none of them (8). This fact may explain the estimated delay of 5 to 14 years in the diagnosis, which contributes to a deterioration of clinical status and a clear impairment in patients' quality of life (8).

Several risk factors are recognized in the setting of HS. Tobacco smoking, for example, is linked with more severe and treatmentresistant disease (9). Due to mechanical irritation, occlusion, and maceration, obesity is a demonstrated contributing factor for HS, and therapy with lithium is also a marked aggravator $(10,11)$. Of note, the role of hormones in the pathophysiology of HS is still controversial, and hormonal fluctuations may merely be acting as a confounding factor (12).

On the other hand, in recent years information has increased about HS-associated diseases, although their significance remains unclear (5). IBD, spondylarthritis, pyoderma gangrenosum, synovitis-acne-pustulosis-hyperostosis-osteitis (SAPHO) syndrome, and Behçet disease are recognized comorbid associations (2, 11). Several endocrinal disorders have also been linked, including diabetes, acromegaly, and Cushing syndrome (2). However, no pathogenic background has yet been suggested (2).

Treatment of HS is influenced by numerous factors, including severity and extension of the disease, comorbidities, and patient age. Among topical agents, $15 \%$ resorcinol may be helpful, and among topical antibiotics clindamycin (0.1\% twice daily) is the only option with a clear benefit $(3,13)$. In moderate to severe cases, systemic therapies are usually required. Systemic antibiotics with a proven benefit include clindamycin (300 mg twice daily) plus rifampicin (300 mg twice daily), clindamycin in monotherapy, or tetracycline (13). According to recent data, isotretinoin is not recommended in the treatment of HS, but acitretin may be an option in selected cases (13). Oral corticosteroids should be reserved for acute flares and for short periods, and intralesional therapy may be especially useful for recalcitrant lesions (13). For moderate to severe HS without response to previous drugs, biological agents may be considered (13). Of note, NSAIDs have no proven benefits in the relief of pain and inflammation (13).

Surgery is the only definite treatment for HS, mainly when there are sinus tracts and scars (3). Radical excision is considered the treatment of choice for these patients, although wide surgical in-

Table 4 | Relationship between HS treatment and year of GP residency vs. consultants.

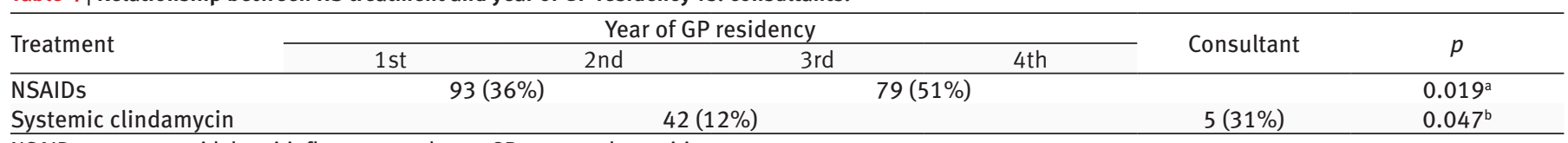

NSAIDs = non-steroidal anti-inflammatory drugs, GP = general practitioner.

${ }^{a}$ Chi-square test

'Fisher's exact test 
tervention may be disfiguring and, ultimately, does not prevent recurrences $(3,13)$. Regarding incision and drainage, this technique results in rapid pain relief, but it is not generally recommended in the treatment of solid inflamed nodules, mostly because there is a high rate of recurrences (3).

In our study, $84 \%$ of the responders considered topical antibiotics and $76 \%$ systemic antibiotics as the treatment of choice for HS. In a previous study by Benhadou et al., Belgian and Danish GPs considered systemic antibiotics the best drug option (14). Drainage was proposed by $59 \%$ of our responders, whereas Belgian and Danish GPs are weaker advocates (20\% and $8 \%$, respectively) of this therapeutic intervention (14).

The majority of GP trainees and consultants recognized HS as an independent entity and confirmed observing several patients per month. However, there seems to be a lack of knowledge concerning disease associations and, most importantly, current therapeutic strategies. During residency, there are few differences in attitudes regarding patients with HS, pointing to gaps in the residency program.

Our study has some limitations. First, there is a potential reporting bias naturally related to the questionnaire-based methodology. Second, there is a difference between the categorized groups, with a small proportion of GP consultants, which may influence the analysis. Finally, there was a $28 \%$ rate of non-responders in our study. There are several possible explanations for this finding: non-responders may not be interested in this issue or they may lack of knowledge about HS and therefore have chosen not to respond.

GPs must play a key role not only in early diagnosis but also in therapeutic decisions and in controlling comorbidities of patients with HS. To us, it seems crucial to promote the development of skills on this issue, so that a referral to dermatology is made correctly and with the appropriate timing whenever first-line measures are insufficient.

\section{References}

1. Alavi A, Lynde C, Alhusayen R, Bourcier M, Delorme I, George R, et al. Approach to the management of patients with hidradenitis suppurativa: a consensus document. J Cutan Med Surg. 2017;21:513-24.

2. Fimmel S, Zouboulis CC. Comorbidities of hidradenitis suppurativa (acne inversa). Dermatoendocrinol. 2010;2:9-16.

3. Saunte DML, Jemec GBE. Hidradenitis suppurativa: advances in diagnosis and treatment. JAMA. 2017;318:2019-32.

4. Desai N, Shah P. High burden of hospital resource utilization in patients with hidradenitis suppurativa in England: a retrospective cohort study using hospital episode statistics. Br J Dermatol. 2017;176:1048-55.

5. Gulliver W, Landells IDR, Morgan D, Pirzada S. Hidradenitis suppurativa: a novel model of care and an integrative strategy to adopt an orphan disease. J Cutan Med Surg. 2018;22:71-7.

6. Parsi KK, Brezinski EA, Lin TC, Li CS, Armstrong AW. Are patients with psoriasis being screened for cardiovascular risk factors? A study of screening practices and awareness among primary care physicians and cardiologists. J Am Acad Dermatol. 2012;67:357-62.

7. Costa-Silva M, Vide J, Lopes S, Azevedo F, Magina S. Psoriasis and comorbidities: general practitioners' awareness. Acta Dermatovenerol Alp Pannonica Adriat. 2018;27:5-7.
8. Jemec GB, Kimball AB. Hidradenitis suppurativa: epidemiology and scope of the problem. J Am Acad Dermatol. 2015;73:S4-7.

9. Garg A, Papagermanos V, Midura M, Strunk A. Incidence of hidradenitis suppurativa among tobacco smokers: a population-based retrospective analysis in the U.S.A. Br J Dermatol. 2018;178:709-14.

10. Witmanowski H, Szychta P, Stepniewski S, Mackiewicz-Wysocka M, CzyzewskaMajchrzak L, Wasilewska A. Acne inversa goes an extra mile than hidradenitis suppurativa. Postepy Dermatol Alergol. 2013;30:255-60.

11. Patil S, Apurwa A, Nadkarni N, Agarwal S, Chaudhari P, Gautam M. Hidradenitis suppurativa: inside and out. Indian J Dermatol. 2018;63:91-8.

12. Karagiannidis I, Nikolakis G, Sabat R, Zouboulis CC. Hidradenitis suppurativa / acne inversa: an endocrine skin disorder? Rev Endocr Metab Disord. 2016;17: 335-41.

13. Zouboulis CC, Desai N, Emtestam L, Hunger RE, Ioannides D, Juhasz I, et al. European $\mathrm{S} 1$ guideline for the treatment of hidradenitis suppurativa / acne inversa. J Eur Acad Dermatol Venereol. 2015;29:619-44.

14. Benhadou F, Theut Riis P, Njimi HH, Jemec GBE, del Marmol V. Hidradenitis suppurativa in general practice: a pilot study. J Gen Pract (Los Angel). 2015:3:207. 